\title{
Entropy production and heat transport in harmonic chains under time dependent periodic drivings
}

\author{
Bruno A. N. Akasaki, Mário J. de Oliveira and C. E. Fiore \\ Universidade de São Paulo, Instituto de Física, Rua do Matão, 1371, 05508-090 São Paulo, SP, Brasil
}

(Dated: November 4, 2019)

\begin{abstract}
Using stochastic thermodynamics, the properties of interacting linear chains subject to periodic drivings are investigated. The systems are described by Fokker-Planck-Kramers equation and exact (explicit) solutions are obtained for periodic drivings as functions of the modulation frequency and strength constants. The limit of long chains is analyzed by means of a protocol in which the intermediate temperatures are self-consistently chosen and the entropy production is decomposed as a sum of two individual contributions, one coming from real baths (placed at extremities of lattice) and other from self-consistent baths. The thermal reservoirs lead to a heat flux according to Fourier's law. Explicit expressions for short chains are derived, whose entropy production is written down as a bilinear function of thermodynamic forces and the associated fluxes, from which Onsager coefficients have been evaluated. A comparison between distinct periodic drivings is also performed.
\end{abstract}

\section{INTRODUCTION}

The description of thermodynamic quantities at the microscopic level gives rise to the stochastic thermodynamics [1-3], in which fluctuations in the thermodynamic fluxes become important. This theory not only allows to reproduce the fundamental concepts of thermodynamics of equilibrium systems but can also be extended for the more general case of nonequilibrium ones. In particular, it shows that stochastic fluxes satisfy general relations such as the Jarzynski equality [4, [5] or/and it predicts the existence of general bounds among thermodynamic fluxes 6,7$]$.

In the last years, the concept of entropy production has played a fundamental role in nonequilibrium statistical physics not only for typifying the irreversibility [8 10], but also for tackling general considerations about efficiency of heat engines [11], the analysis of (irreversible) phase transition portraits 12 14], thermodynamic uncertainties relations [6, 7] and others. A fundamental relation for the entropy production comes from simple entropic arguments in which the system is coupled to a thermal reservoir. Its time entropy variation $d S / d t$ is the difference of two terms

$$
\frac{d S}{d t}=\Pi(t)-\Phi(t)
$$

where $\Pi(t)$ is the entropy production rate and $\Phi(t)$ is the entropy flux rate from/to the system to/from the environment. Since the environment works a subsystem in equilibrium, $\Pi(t)$ corresponds to the entropy produced inside the system. Eq. (11) implies that all entropy spontaneously produced (by the system) has to be delivered to the environment in the steady state regime. When the system is in thermal equilibrium, it follows that $\Pi_{s}=\Phi_{s}=0$, whereas $\Pi_{s}=\Phi_{s}>0$ out of the equilibrium regime. Thereby entropy production discerns equilibrium and nonequilibrium systems, since in the latter case it is continuously produced. In such case, the steady entropy production rate can alternatively be evaluated through the calculation of the steady entropy flux
$\Phi_{s}$.

The thermodynamic properties of Markovian systems have been extensively studied in the last years, including those described by master [8 10, 15, 16] and FokkerPlanck equations [17 22]. A special recent attention has been devoted to periodically driven systems $23-26]$. In part because their thermodynamic properties can be experimentally accessible [23, 27-44]. In addition, some of their remarkable features, such as a general description in the linear regime (Onsager coefficients and general reciprocal relations can be achieved), the existence of uncertainties constraints leading to existence of bounds among macroscopic averages and other features have been extensively studied. However, the more general case of interacting particles subject to time periodic drivings has not been studied thoroughly.

In this paper, we fill this gap by investigating the thermodynamic properties of interacting chains of Brownian particles subject to (time dependent) periodic forces and temperature drivings. Exact expressions for the thermodynamic quantities, including the dissipated heat, entropy production, heat flux and others are obtained. The limits of short and long particle chains are thoroughly investigated. For the latter case, intermediate temperatures are self-consistently chosen through a protocol taking into account an inner entropy production source. This is meaningfully different from the original approach by Bosterli et al. [47], in which no heat flux is exchanged among the particles and self-consistent baths. Thus, our approach provides us not only to analyze the role of two distinct sources of dissipation, but also establishing which contribution dominates in the limits of short and long chains. Two main findings can be drawn: For the case of two interacting particles, the entropy production is derived as a bilinear function of fluxes and forces for both drivings in forces and temperatures, whose associated Onsager coefficients depend on the interaction parameters and frequency driving. The entropy production of long particle chains can be splitted in two terms: one coming from the thermal reservoirs and the other from the self-consistent ones. Other remarkable features, such 
as the effect of a phase difference (a lag) between external forces are investigated and the condition for maximum/minimum entropy production is found to depend only on the temperature reservoirs and frequency driving, irrespective the interaction strength between particles.

This paper is organized as follows: In Sec. II we describe the theoretical background and exact solution for time dependent drivings is evaluated in Sec. III. In Sec. IV and V, the two and several particles cases are analyzed, respectively. Conclusions are discussed in Sec. VI.

\section{FOKKER-PLANCK-KRAMERS EQUATION}

We consider a set of $N$ interacting particles with equal masses $m$, in which the $i$-th particle evolves in time according to the following set of coupled Langevin equations

$$
\frac{d v_{i}}{d t}=f_{i}^{*}-\gamma v_{i}+\mathcal{F}_{i}(t)
$$

and

$$
\frac{d x_{i}}{d t}=v_{i}
$$

with $x_{i}$ denoting its position with velocity $v_{i}=d x_{i} / d t$, respectively, whereas $\gamma$ is the dissipation constant. Here $f_{i}^{*}$ stands for the force acting to the $i$-th particle, which is assumed to be decomposed as the sum of a time dependent term $f_{i}^{e x t}(t)$ plus a term $f_{i}$ depending only on the positions. Thus, $f_{i}$ can be written as the derivative of the potential energy $V, f_{i}=-\partial V / \partial x_{i}$. The stochastic force $\mathcal{F}_{i}(t)$ accounts for the interaction between particle $i$ and the environment and satisfies the properties

$$
\left\langle\mathcal{F}_{i}(t)\right\rangle=0
$$

and

$$
\left\langle\mathcal{F}_{i}(t) \mathcal{F}_{j}\left(t^{\prime}\right)\right\rangle=2 \gamma T_{i} \delta_{i j} \delta\left(t-t^{\prime}\right)
$$

respectively, where $T_{i}>0$ is distinct for each particle. Let $P(x, v, t) \equiv P\left(x_{1}, . ., x_{N}, v_{1}, \ldots, v_{N}, t\right)$ be the joint probability distribution at time $t$, where $x$ and $v$ denote the collection of particle positions $x_{i}$ and velocities $v_{i}$, respectively. Its time evolution is described by the FokkerPlanck-Kramers (FPK) equation [3, 9, 18]

$$
\frac{\partial P}{\partial t}=-\sum_{i}\left(v_{i} \frac{\partial P}{\partial x_{i}}+\left[f_{i}+f_{i}^{e x t}(t)\right] \frac{\partial P}{\partial v_{i}}+\frac{\partial J_{i}}{\partial v_{i}}\right)
$$

where

$$
J_{i}=-\gamma v_{i} P-\frac{\gamma k_{\mathrm{B}} T_{i}}{m} \frac{\partial P}{\partial v_{i}}
$$

If the temperatures of all particles $T_{i}$ are the same and the external forces are null, the probability distribution approaches for large times the Gibbs equilibrium distribution,

$$
P^{e}(x, v)=\frac{1}{Z} e^{-E / k_{\mathrm{B}} T}
$$

where $E=m v^{2} / 2+V$ is the energy of the system. This result shows that the FPK Eq. (6) indeed describes the contact of a system with a heat reservoir at a temperature $T$. On the other hand, this will not be the case of the system in contact with distinct reservoirs and/or when it is subject to time oscillating forces or temperatures. In such case, the system dissipates heat and continuously produce entropy.

From the FK equation, the time variation of the energy $U=\langle E\rangle$ reads

$$
\frac{d U}{d t}=-\sum_{i=1}^{N}\left(\Phi_{\mathrm{q}}^{(i)}+\Phi_{\mathrm{w}}^{(i)}\right)
$$

where the heat flux $\Phi_{\mathrm{q}}^{(i)}$ from the system to the environment (thermal bath) is expressed as [9, 18]

$$
\Phi_{\mathrm{q}}^{(i)}=\gamma\left(m\left\langle v_{i}^{2}\right\rangle-k_{\mathrm{B}} T_{i}\right)
$$

whose first and second terms can be understood as the heating power and the power of heat losses, respectively. The term $\Phi_{\mathrm{w}}^{(i)}$ can be interpreted as the work per unity of time given by

$$
\Phi_{\mathrm{w}}^{(i)}=-m\left\langle v_{i}\right\rangle f_{i}^{e x t}(t) .
$$

In the absence of external forces all heat flux comes from/goes to the thermal bath.

The entropy $S$ of the system is determined from the Gibbs expression

$$
S=-k_{\mathrm{B}} \int P \ln P d x d v .
$$

From the FPK equation, one finds that its time derivative has the form of Eq. (11), where the first is identified as the rate of entropy production given by [9, 18]

$$
\Pi=\frac{m k_{\mathrm{B}}}{\gamma T_{i}} \sum_{i=1}^{N} \int \frac{J_{i}^{2}}{P} d x d v .
$$

Note that $\Pi \geq 0$ (as expected). Conversely, the second term corresponds to the flux of entropy given by

$$
\Phi=-\sum_{i=1}^{N} \frac{m k_{\mathrm{B}}}{T_{i}} \int v_{i} J_{i} d x d v
$$

or even rewritten as

$$
\Phi=k_{\mathrm{B}} \sum_{i=1}^{N} \frac{\Phi_{\mathrm{q}}^{(i)}}{T_{i}} .
$$

As mentioned previously, Eq. (15) can be alternatively used for evaluated the steady production of entropy, since it depends only on averages $\left\langle v_{i}^{2}\right\rangle$ and on the temperatures $T_{i}$. 


\section{EXACT SOLUTION FOR TIME DEPENDENT EXTERNAL FORCES}

For simplifying matters, from now on we shall adopt $k_{\mathrm{B}}=1$. Except in Sec. IVB, all analysis will restrict to the case of a chain of $N$ particles interacting to its nearest neighbors subject to an external force. The expression for the force of $i$-th particle $f_{i}^{*}$ then reads

$$
\begin{aligned}
& f_{i}^{*}=-\frac{k}{m}\left(x_{i}-x_{i+1}\right)-\frac{k^{\prime}}{m} x_{i}+f_{i}^{\text {ext }}(t), \\
& f_{i}^{*}=-\frac{k}{m}\left(x_{i}-x_{i-1}\right)-\frac{k^{\prime}}{m} x_{i}+f_{i}^{\operatorname{ext}}(t),
\end{aligned}
$$

for particles placed at extremities, $i=1$ and $N$, respectively, and

$$
f_{i}^{*}=-\frac{k}{m}\left(2 x_{i}-x_{i-1}-x_{i+1}\right)-\frac{k^{\prime}}{m} x_{i}+f_{i}^{\text {ext }}(t),
$$

for the intermediate ones. Above expressions can be conveniently rewritten as

$$
\begin{aligned}
& f_{i}^{*}=-K x_{i}+L x_{i+1}+f_{i}^{\text {ext }}(t), \\
& f_{i}^{*}=-K x_{i}+L x_{i-1}+f_{i}^{\text {ext }}(t),
\end{aligned}
$$

and, for $i=1$ and $i=N$, respectively, and

$$
f_{i}^{*}=-(K+L) x_{i}+L\left(x_{i+1}+x_{i-1}\right)+f_{i}^{\text {ext }}(t),
$$

respectively, where $L=k / m$ and $K=\left(k+k^{\prime}\right) / m$ for $i \neq 1$ and $i \neq N$.

The time evolution of a generic average of type $\langle g\rangle=$ $\int g(x, y) P(x, v, t) d x d v$ is obtained through expression

$$
\frac{d}{d t}\langle g\rangle=\int g(x, y) \frac{\partial P}{\partial t} d x d v
$$

and by inserting Eq. (6) in Eq. (22) and performing appropriate partial integrations, an explicit equation for the time evolution of $\langle g\rangle$ is evaluated in terms of correlations associated to the positions and velocities. Due to the time dependence on the external forces, the evaluation of averages like $\langle g\rangle$ becomes cumbersome. However, the calculations become quite simpler by rewriting the motion equations in terms of their associate covariances. For instance, let us take for example a generic average $\langle g\rangle=$ $\left\langle x_{i}^{l} x_{j}^{m}\right\rangle$ (with $l \geq 1$ and $m \geq 1$ ) with covariance given by $\left\langle x_{i}^{l} x_{j}^{m}\right\rangle_{T} \equiv\left\langle x_{i}^{l} x_{j}^{m}\right\rangle-\left\langle x_{i}^{l}\right\rangle\left\langle x_{j}^{m}\right\rangle$. Unlike the time evolution of $\left\langle x_{i}^{l} x_{j}^{m}\right\rangle$, the time equation for $d\left\langle x_{i}^{l} x_{j}^{m}\right\rangle_{T} / d t=$ $d\left\langle x_{i}^{l} x_{j}^{m}\right\rangle / d t-\left\langle x_{j}^{m}\right\rangle d\left\langle x_{i}^{l}\right\rangle / d t-\left\langle x_{i}^{l}\right\rangle d\left\langle x_{j}^{m}\right\rangle / d t$ does not depend explicitly on $t$. Since the equations for all covariances are linear and time independent, the exact solution is possible for all system sizes $N$. Finally, having the averages $\left\langle v_{i}^{2}\right\rangle_{T}$ and $\left\langle v_{i}\right\rangle^{2}$, the entropy flux can be directly evaluated from the usage of Eqs. (10) and (15).
Below we derive explicit expressions for distinct covariances between the $i-$ and $i+1$-th particles for a generic chain of $N$ sites.

$$
\begin{gathered}
\frac{d}{d t}\left\langle x_{i}^{2}\right\rangle_{T}=2\left\langle v_{i} x_{i}\right\rangle_{T}, \\
\frac{d}{d t}\left\langle x_{i} x_{i+1}\right\rangle_{T}=\left\langle v_{i} x_{i+1}\right\rangle_{T}+\left\langle v_{i} x_{i+1}\right\rangle_{T}, \\
\frac{d}{d t}\left\langle x_{i} v_{i}\right\rangle_{T}=\left\langle v_{i}^{2}\right\rangle_{T}-K\left\langle x_{i}^{2}\right\rangle_{T}+L\left\langle x_{i} x_{i+1}\right\rangle_{T}-\gamma\left\langle x_{i} v_{i}\right\rangle_{T}, \\
\frac{d}{d t}\left\langle x_{i} v_{i+1}\right\rangle_{T}=\left\langle v_{i} v_{i+1}\right\rangle_{T}-K\left\langle x_{i} x_{i+1}\right\rangle_{T}+L\left\langle x_{i}^{2}\right\rangle_{T} \\
-\gamma\left\langle x_{i} v_{i+1}\right\rangle_{T}, \\
\frac{d}{d t}\left\langle v_{i}^{2}\right\rangle_{T}=-2 K\left\langle v_{i} x_{i}\right\rangle_{T}+2 L\left\langle x_{i+1} v_{i}\right\rangle_{T}-2 \gamma\left\langle v_{i}^{2}\right\rangle_{T}+\Gamma_{i}, \\
\frac{d}{d t}\left\langle v_{i} v_{i+1}\right\rangle_{T}=-K\left\langle x_{i} v_{i+1}\right\rangle_{T}+L\left\langle x_{i+1} v_{i+1}\right\rangle_{T} \\
-K\left\langle x_{i+1} v_{i}\right\rangle_{T}+L\left\langle x_{i} v_{i}\right\rangle_{T}-2 \gamma\left\langle v_{i} v_{i+1}\right\rangle_{T}
\end{gathered}
$$

Here we introduced the rescaled temperature $\Gamma_{i}$ defined by $\Gamma_{i}=2 \gamma T_{i} / m$ and thereby for fixed $\Gamma_{i}$ 's, the achievement of $\left\langle v_{i}^{2}\right\rangle_{T}$ 's reduces to systems of linear equations.

The time evolution of single averages $\left\langle v_{i}\right\rangle$ and $\left\langle x_{i}\right\rangle$ are also required for obtaining $\left\langle v_{i}^{2}\right\rangle$, whose expressions read

$\frac{d}{d t}\left\langle v_{i}\right\rangle=-(K+L)\left\langle x_{i}\right\rangle+L\left(\left\langle x_{i+1}\right\rangle+\left\langle x_{i-1}\right\rangle\right)-\gamma\left\langle v_{i}\right\rangle+f_{i}^{e x t}(t)$,

for $i \neq 1, N$ and

$$
\begin{aligned}
& \frac{d}{d t}\left\langle v_{i}\right\rangle=-K\left\langle x_{i}\right\rangle+L\left\langle x_{i+1}\right\rangle-\gamma\left\langle v_{i}\right\rangle+f_{i}^{e x t}(t), \\
& \frac{d}{d t}\left\langle v_{i}\right\rangle=-K\left\langle x_{i}\right\rangle+L\left\langle x_{i-1}\right\rangle-\gamma\left\langle v_{i}\right\rangle+f_{i}^{e x t}(t),
\end{aligned}
$$

for $i=1$ and $N$, respectively and from Eq. (3), the time evolution of $\left\langle x_{i}\right\rangle$ reads

$$
\frac{d}{d t}\left\langle x_{i}\right\rangle=\left\langle v_{i}\right\rangle
$$

Although the previous procedure does not depend on the shape of external forces, from now on we will restrict our analysis to harmonic external forces given by $f_{i}^{e x t}(t)=f_{0 i} \cos (\omega t+\phi)$, with $\omega$ and $\phi$ being its frequency and phase difference (lag), respectively. By assuming that each $\left\langle x_{i}\right\rangle$ has solution of type $\left\langle x_{i}\right\rangle=$ $A_{0 i}+A_{1 i} \cos \omega t+A_{2 i} \sin \omega t,\left\langle v_{i}\right\rangle$ becomes $\left\langle v_{i}\right\rangle=$ $\omega\left[A_{2 i} \cos (\omega t)-A_{1 i} \sin (\omega t)\right]$. By inserting above solutions in Eqs. (29) [or Eq. (30)/(31)] and (32), the coefficients $A_{1 i}$ and $A_{2 i}$ are obtained. 


\section{TWO PARTICLES CASE}

\section{A. Oscillating forces}

In the first application we analyze a chain of two interacting particles subjected to harmonic forces without phase difference (lagless case, $\phi=0$ ). From the solution of linear set of equations described in Sec. III, we obtain the following expressions for the covariances:

$$
\left\langle v_{1}^{2}\right\rangle_{T}=\frac{\Gamma_{1}+\Gamma_{2}}{4 \gamma}+\frac{K \gamma\left(\Gamma_{1}-\Gamma_{2}\right)}{4\left(L^{2}+K \gamma^{2}\right)},
$$

and

$$
\left\langle v_{2}^{2}\right\rangle_{T}=\frac{\Gamma_{1}+\Gamma_{2}}{4 \gamma}-\frac{K \gamma\left(\Gamma_{1}-\Gamma_{2}\right)}{4\left(L^{2}+K \gamma^{2}\right)}
$$

The entropy flux $\Phi(t)$ can be splitted in two parts,

$$
\Phi(t)=\Phi_{T}+\Phi_{f}(t)
$$

where $\Pi_{T}=\Phi_{T}$ (in the steady state regime) and $\Phi_{f}(t)$ read

$$
\Phi_{T}=\frac{2 \gamma^{2}}{\Gamma_{1}}\left\langle v_{1}^{2}\right\rangle_{T}+\frac{2 \gamma^{2}}{\Gamma_{2}}\left\langle v_{2}^{2}\right\rangle_{T}-2 \gamma
$$

and

$$
\Phi_{f}(t)=\frac{2 \gamma^{2}}{\Gamma_{1}}\left\langle v_{1}\right\rangle^{2}+\frac{2 \gamma^{2}}{\Gamma_{2}}\left\langle v_{2}\right\rangle^{2}
$$

respectively. Above expressions can be simplified, acquiring the following form

$$
\Pi_{T}=\frac{\gamma L^{2}}{2\left(L^{2}+K \gamma^{2}\right)} \frac{\left(\Gamma_{1}-\Gamma_{1}\right)^{2}}{\Gamma_{1} \Gamma_{2}}
$$

and

$$
\Phi_{f}(t)=2 \gamma^{2} \omega^{2} \sum_{i}\left(\frac{\left(A_{2 i} \cos \omega t-A_{1 i} \sin \omega t\right)^{2}}{\Gamma_{i}}\right)
$$

respectively, whose coefficients $A_{1 i}$ and $A_{2 i}$ are shown in Appendix $\mathrm{A}$. Note that $\Pi_{T}$ depends solely on the difference of temperatures and are similar to the case with no external forces [18], whereas $\Phi_{f}(t)$ is related to the time dependent forces. By evaluating $\Phi_{f}(t)$ over a period cycle, we arrive at the following expression for $\bar{\Pi}=\frac{\omega}{2 \pi} \int_{0}^{2 \pi / \omega} \Phi_{f}(t) d t$

$$
\bar{\Pi}=\frac{\gamma^{2} \omega^{2}\left[\Gamma_{1}\left(A_{12}^{2}+A_{22}^{2}\right)+\Gamma_{2}\left(A_{11}^{2}+A_{21}^{2}\right)\right]}{\Gamma_{1} \Gamma_{2}} .
$$

By substituting the expressions for $A_{1 i}$ 's and $A_{2 i}$ 's we finally arrive at the following expression:

$\bar{\Pi}=\frac{\gamma^{2} \omega^{2}\left\{f_{01}^{2}\left[\Gamma_{2}\left(\gamma^{2} \omega^{2}+\left(K-\omega^{2}\right)^{2}\right)+L^{2} \Gamma_{1}\right]+2 f_{01} f_{02} L\left(K-\omega^{2}\right)\left(\Gamma_{1}+\Gamma_{2}\right)+f_{02}^{2}\left[\Gamma_{1}\left(\gamma^{2} \omega^{2}+\left(K-\omega^{2}\right)^{2}\right)+L^{2} \Gamma_{2}\right]\right\}}{\Gamma_{1} \Gamma_{2}\left[\gamma^{2} \omega^{2}+\left(K+L-\omega^{2}\right)^{2}\right]\left[\gamma^{2} \omega^{2}+\left(-K+L+\omega^{2}\right)^{2}\right]}$.

This is the one of main results of the paper, and we pause to make some few comments: First, from Eq. (40) it follows that $\bar{\Pi}$ is always greater than 0 , vanishing when $f_{01}=f_{02}=0$ and $/$ or $\omega=0$. Second, in the limit of slow or fast oscillations, $\omega<<1$ or $\omega>>1, \bar{\Pi}$ behaves as

$$
\bar{\Pi} \sim \frac{\gamma^{2}\left[\Gamma_{1}\left(f_{01} K+f_{02} L\right)^{2}+\Gamma_{2}\left(f_{01} L+f_{02} K\right)^{2}\right] \omega^{2}}{\Gamma_{1} \Gamma_{2}\left(K^{2}-L^{2}\right)^{2}}
$$

and

$$
\bar{\Pi} \sim\left(\frac{f_{01}^{2} \Gamma_{2}+f_{02}^{2} \Gamma_{1}}{\Gamma_{1} \Gamma_{2}}\right) \frac{\gamma^{2}}{\gamma^{2}+\omega^{2}},
$$

respectively, implying that $\bar{\Pi}$ vanishes as $\omega^{2}$ and $1 / \omega^{2}$ for low and large periods, respectively. Third, when the interaction between particles is "weak", $k<<k^{\prime}, \bar{\Pi}$ re- duces to the single forced harmonic oscillator expression:

$$
\bar{\Pi} \sim \frac{\gamma^{2} \omega^{2}\left(f_{01}^{2} \Gamma_{2}+f_{02}^{2} \Gamma_{1}\right)}{\Gamma_{1} \Gamma_{2}\left[\gamma^{2} \omega^{2}+(K-\omega)^{2}\right]},
$$

acquiring the simpler form

$$
\bar{\Pi} \sim \frac{\gamma^{2} \omega^{2} f_{01}^{2}}{\Gamma_{1}\left[\gamma^{2} \omega^{2}+(K-\omega)^{2}\right]},
$$

as $\Gamma_{1}=\Gamma_{2}$ and $f_{01}=f_{02}$. Fourth and last, in the strong coupling regime, $k>>k^{\prime}$ and $k / m>>\omega^{2}$ (or equivalently $L \approx K$ and $L>>\omega^{2}$ ), $\bar{\Pi}$ becomes

$$
\bar{\Pi} \sim\left(\frac{\gamma^{2}}{\gamma^{2}+\omega^{2}}\right)\left(\frac{\Gamma_{1}+\Gamma_{2}}{\Gamma_{1} \Gamma_{2}}\right)\left(f_{01}+f_{02}\right)^{2},
$$

which is independent on strength oscillator parameters $K$ and $L$. 


\section{Bilinear form and Onsager coefficients}

The shapes of Eqs. (38) and (41) show that the entropy production components can be written as flux-times-force expressions $\Pi_{T}=\mathcal{J}_{T} f_{T}$ and

$$
\bar{\Pi}=\mathcal{J}_{1}^{f} f_{01}+\mathcal{J}_{2}^{f} f_{02}
$$

respectively, where the forces $f_{T}=1 / \Gamma_{1}-1 / \Gamma_{2}$ and $f_{0 i(j)}$ have associated fluxes $\mathcal{J}_{T}, \mathcal{J}_{1}^{f}$ and $\mathcal{J}_{2}^{f}$ given by

$$
\mathcal{J}_{T}=\frac{\Gamma_{1} \Gamma_{2} \gamma L^{2}}{2\left(L^{2}+K \gamma^{2}\right)}\left(\frac{1}{\Gamma_{1}}-\frac{1}{\Gamma_{2}}\right)
$$

and

$$
\mathcal{J}_{1}^{f}=L_{11} f_{01}+L_{12} f_{02}, \quad \text { and } \quad \mathcal{J}_{2}^{f}=L_{21} f_{01}+L_{22} f_{02} \text {, }
$$

respectively. The bilinear form for $\bar{\Pi}$ provides to identify the terms $L_{11}$ and $L_{12}$ as the associated Onsager coefficients given by

$$
L_{11}=\frac{\gamma^{2} \omega^{2}\left[\Gamma_{2}\left(\gamma^{2} \omega^{2}+\left(K-\omega^{2}\right)^{2}\right)+L^{2} \Gamma_{1}\right]}{\Gamma_{1} \Gamma_{2}\left[\gamma^{2} \omega^{2}+\left(K+L-\omega^{2}\right)^{2}\right]\left[\gamma^{2} \omega^{2}+\left(-K+L+\omega^{2}\right)^{2}\right]},
$$

and

$$
L_{12}=\frac{L\left(K-\omega^{2}\right)\left(\Gamma_{1}+\Gamma_{2}\right)}{\Gamma_{1} \Gamma_{2}\left[\gamma^{2} \omega^{2}+\left(K+L-\omega^{2}\right)^{2}\right]\left[\gamma^{2} \omega^{2}+\left(-K+L+\omega^{2}\right)^{2}\right]},
$$

respectively. Analogous expressions are hold valid for $L_{21}$ and $L_{22}$ by exchanging $1 \leftrightarrow 2$. Note that $L_{11} \geq 0$ and $L_{22} \geq 0$ (as expected). The non-negativity of the entropy production also requires that $4 L_{11} L_{22}-\left(L_{12}+L_{21}\right)^{2} \geq 0$. To verify this, let us consider $\Gamma_{2}=r \Gamma_{1}$ with $r$ being an arbitrary (non negative) real number. Such above inequality is always satisfied, since the term

$$
\frac{\left[\gamma^{2} \omega^{2}+\left(K-\omega^{2}\right)^{2}+L^{2} r\right]\left[r\left(\gamma^{2} \omega^{2}+\left(K-\omega^{2}\right)^{2}\right)+L^{2}\right]}{L^{2}(r+1)^{2}\left(K-\omega^{2}\right)^{2}},
$$

is greater than $1 / 4$ for all values of $r, K, \omega$ and $\gamma$.

\section{Phase difference between harmonic forces}

Here we extend the results from previous section but taking into account a phase difference between external forces $f_{1}^{e x t}(t)$ and $f_{2}^{e x t}(t)$. More specifically, $f_{1}^{e x t}(t)$ has the same expression as previously, but $f_{2}^{e x t}(t)$ now reads $f_{2}^{\text {ext }}(t)=f_{02} \cos (\omega t+\phi)$. By repeating aforedescribed procedures, we assume that $\left\langle v_{i}\right\rangle=$ $w\left(C_{2 i} \cos \omega t-C_{1 i} \sin \omega t\right)$, whose coefficients $C_{1 i}$ and $C_{2 i}$ are decomposed in two parts: $C_{1 i}=A_{1 i}+B_{1 i}(\phi)$, whose $A_{1 i}$ and $A_{2 i}$ are the same as Eqs. (A1) and (A2) and the dependence on the phase difference appears only in $B_{1 i}$ and $B_{2 i}$. We then arrive at the following expression for the steady entropy production $\bar{\Pi}$

$$
\bar{\Pi}=\frac{\gamma^{2} \omega^{2}\left[\Gamma_{1}\left(C_{12}^{2}+C_{22}^{2}\right)+\Gamma_{2}\left(C_{11}^{2}+C_{21}^{2}\right)\right]}{\Gamma_{1} \Gamma_{2}} .
$$

Above expression is quite similar to Eq. (40) and as in the lagless case, it has three terms with first and third terms being identical to Eq. (41) and the phase difference dependence appearing only in middle term and given by

$$
\left[\frac{2 f_{01} f_{02} L\left[\gamma \omega\left(\Gamma_{2}-\Gamma_{1}\right) \sin \phi+\left(K-\omega^{2}\right)\left(\Gamma_{1}+\Gamma_{2}\right) \cos \phi\right]}{\Gamma_{1} \Gamma_{2}\left(\gamma^{2} \omega^{2}+\left(K+L-\omega^{2}\right)^{2}\right)\left(\gamma^{2} \omega^{2}+\left(-K+L+\omega^{2}\right)^{2}\right)}\right] .
$$

Note that the it reduces to the middle term from Eq. (41) when $\phi=0$. The position of the maximum and minimum in the average entropy production fulfills the above relation

$$
\phi=\tan ^{-1}\left[\frac{\gamma \omega\left(\Gamma_{2}-\Gamma_{1}\right)}{\left(K-\omega^{2}\right)\left(\Gamma_{1}+\Gamma_{2}\right)}\right] .
$$

Note that $\phi$ depends only on the signs of both $\Gamma_{2}-\Gamma_{1}$ and $K-\omega^{2}$ and it is independent on $L$. In particular, in the regime of $\Gamma_{2}>>\Gamma_{1}\left(\Gamma_{2}<<\Gamma_{1}\right), \phi$ is independent on $\Gamma_{i}$ 's, reading $\pm \gamma \omega /\left(K-\omega^{2}\right)$. Conversely, for fast and slow oscillations, it approaches to zero as $\gamma\left(\Gamma_{1}-\Gamma_{2}\right) / K \omega\left(\Gamma_{2}+\right.$ $\left.\Gamma_{1}\right)$ and $\gamma \omega\left(\Gamma_{2}-\Gamma_{1}\right) / K\left(\Gamma_{2}+\Gamma_{1}\right)$, respectively. Fig. 1 plots $\bar{\Pi}$ versus $\phi$ for distinct set of values of $\omega$ and $\Gamma_{i}$ 's. Note that the maxima of mean entropy production yields at $\phi \sim 0(\pi)$ for small (large) values of $\omega$ and $\phi \rightarrow \pi / 2$ when $\omega \rightarrow \sqrt{K}$. The dependence of extremes clearly follows theoretical predictions from Eq. (54) (see e.g. panels $(a)$ and $(b)$ in Fig. 1).

\section{B. Oscillating temperatures}

The investigation of systems under oscillating temperature has been reported in several works [27 44], which provides a way of measuring the heat capacity experimentally. Here we intend to verify the nonequilibrium properties of a chain of two interacting particles under time oscillating temperatures. For simplicity, we consider external forces absent. The entropy production can also be evaluated straightforwardly from Eq. (15), but instead the temperature $\Gamma_{i}(t)$ of the each reservoir is now time dependent and given by $\Gamma_{i}(t)=\Gamma_{0 i}+F_{T_{i}} \cos (\omega t)$, with $\Gamma_{0 i}$ and $F_{T_{i}}$ being the reference temperature and the strength of temperature driving, respectively.

Although such problem is exactly solvable [see e.g. Fig. 2 (b)] and reduces to the findings from Ref. [19] when $\Gamma_{01}=\Gamma_{02}$ and $L=0$, the expression for the steady entropy production is much more complex than previous cases and involves many terms related to distinct powers of interaction parameters $K, L$ and driving frequency $\omega$. For this reason, our analysis will be carried out close to equilibrium regime, in which a linear treatment can 

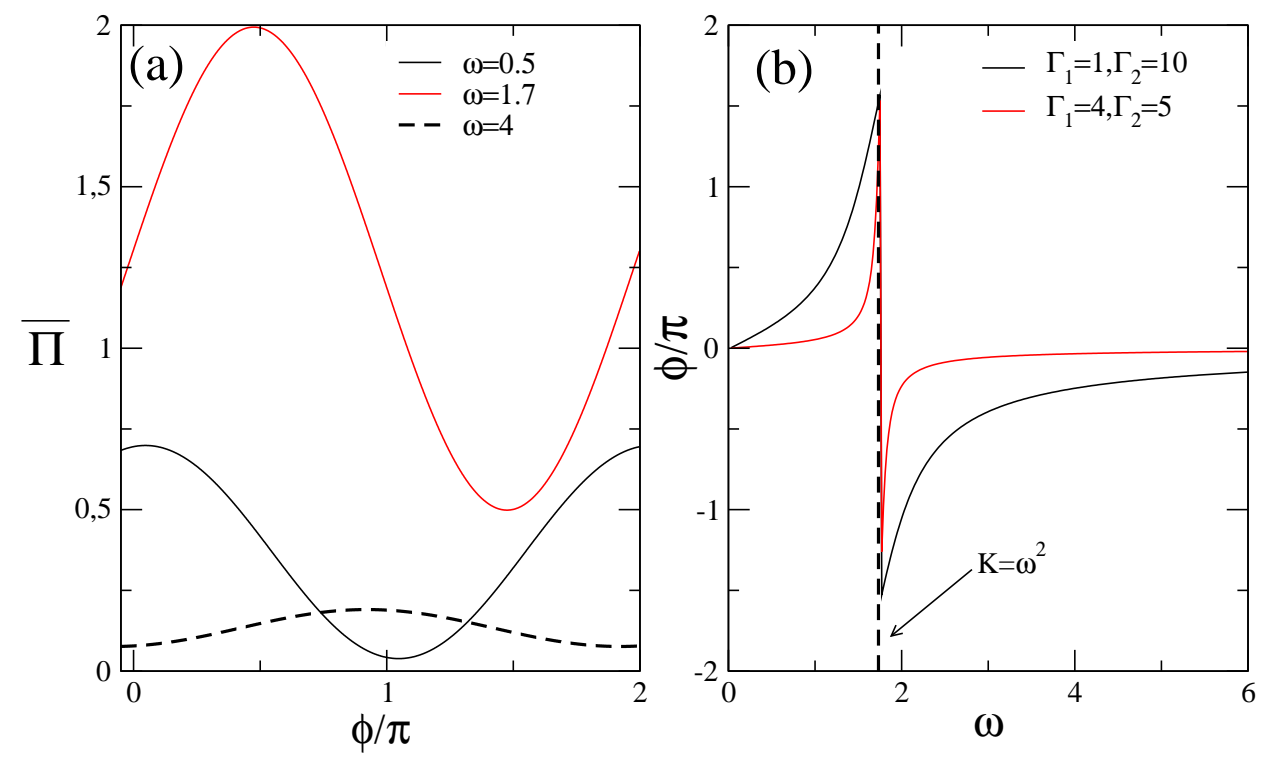

FIG. 1: For distinct frequencies $\omega$ 's, panel $(a)$ depicts the average entropy production $\bar{\Pi}$ versus the phase difference $\phi$ for $\Gamma_{1}=1, \Gamma_{2}=10, K=3$ and $\gamma=1$. For $K=3$ and $\gamma=1$ and distinct sets of $\Gamma_{1}$ and $\Gamma_{2}$, panel $(b)$ shows the positions $\phi$ of maximum/minimum of entropy production $\bar{\Pi}$ versus $\omega$.

be performed. More specifically, we take both reference temperatures to be equal $\Gamma_{01}=\Gamma_{02}=\Gamma_{0}$ and the driving strengths are low $F_{T_{i}}<<\Gamma_{0}$. In such case, the entropy production $\bar{\Pi}$ can also be written down in the bilinear form $\bar{\Pi}=\mathcal{J}_{T_{1}} F_{T_{1}}+\mathcal{J}_{T_{2}} F_{T_{2}}$, where the fluxes $\mathcal{J}_{T_{1}}$ and $\mathcal{J}_{T_{2}}$ read

$$
\mathcal{J}_{T_{1}}=L_{T_{1}, T_{1}} F_{T_{1}}+L_{T_{1}, T_{2}} F_{T_{2}}
$$

and

$$
\mathcal{J}_{T_{2}}=L_{T_{2}, T_{1}} F_{T_{1}}+L_{T_{2}, T_{2}} F_{T_{2}}
$$

respectively, where $L_{T_{i}, T_{j}}$ are the associated Onsager coefficients given by

$$
L_{T_{1}, T_{1}}=\frac{\gamma}{2 \Gamma_{0}^{2}}\left(\frac{\sum_{\ell=0}^{9} B_{\ell} \omega^{2 \ell}}{\sum_{\ell=0}^{9} G_{\ell} \omega^{2 \ell}}\right)
$$

and

$$
L_{T_{1}, T_{2}}=\frac{-4 \gamma^{3} L^{2}}{\Gamma_{0}^{2}}\left(\frac{\sum_{\ell=0}^{6} A_{\ell} \omega^{2 \ell}}{\sum_{\ell=0}^{9} G_{\ell} \omega^{2 \ell}}\right),
$$

respectively, where $L_{T_{1}, T_{1}}=L_{T_{2}, T_{2}}$ and $L_{T_{1}, T_{2}}=L_{T_{2}, T_{1}}$ and coefficients $A_{i}$ 's, $B_{i}$ 's and $G_{i}$ 's depend solely on the parameters $\gamma$ and $L=2 K$ and are listed in the Appendix C

We pause again to make some comments: First, in the limit of slow and fast frequencies, $\bar{\Pi}$ approaches to the following expressions

$$
\bar{\Pi} \sim \frac{4 \gamma^{3} L^{2} A_{0}}{\Gamma_{0}^{2} G_{0}}\left(F_{T_{1}}-F_{T_{2}}\right)^{2},
$$

and

$$
\bar{\Pi} \sim \frac{\gamma}{2 \Gamma_{0}^{2}}\left(F_{T_{1}}^{2}+F_{T_{2}}^{2}\right),
$$

respectively. They contrast with the oscillating forced case, since are independent on $\omega$ and different from zero in both extreme cases. Whenever it depends on $L$ for low oscillations, the entropy production is independent on the coupling for fast oscillations. Finally, for strong interaction strength, $L>>1$ and $L>>\omega^{2}, \bar{\Pi}$ reads

$$
\bar{\Pi} \sim \frac{\gamma}{4 \Gamma_{0}^{2}\left(\gamma^{2}+\omega^{2}\right)}\left[\gamma^{2}\left(F_{T_{1}}-F_{T_{2}}\right)^{2}+2 \omega^{2}\left(F_{T_{1}}^{2}+F_{T_{2}}^{2}\right)\right],
$$

which is also independent on $L$. We close this section by comparing, in Fig. $2(a)$ and $(b)$ the steady entropy production behaviors versus the frequency driving $\omega$ for both oscillating and temperature forces (obtained from the exact solution). They exhibit meaningfully different dependence on $\omega$, even for extreme $\omega$. Whenever $\bar{\Pi}$ vanishes for $\omega<<1$ and $\omega>>1$ in the case of time oscillating forces, it reaches constant values for temperature drivings, in accordance with asymptotic expressions Eqs. (59) and (60), respectively, obtained from the linear regime approximation.

\section{MORE THAN TWO PARTICLES}

In this section we present the main results for long chains of oscillators, in which the difference of temperature between particles placed at the extremities is responsible for a transport of heat following Fourier's law. More concretely, it states that the heat current is proportional to the inverse of the length of the chain given 

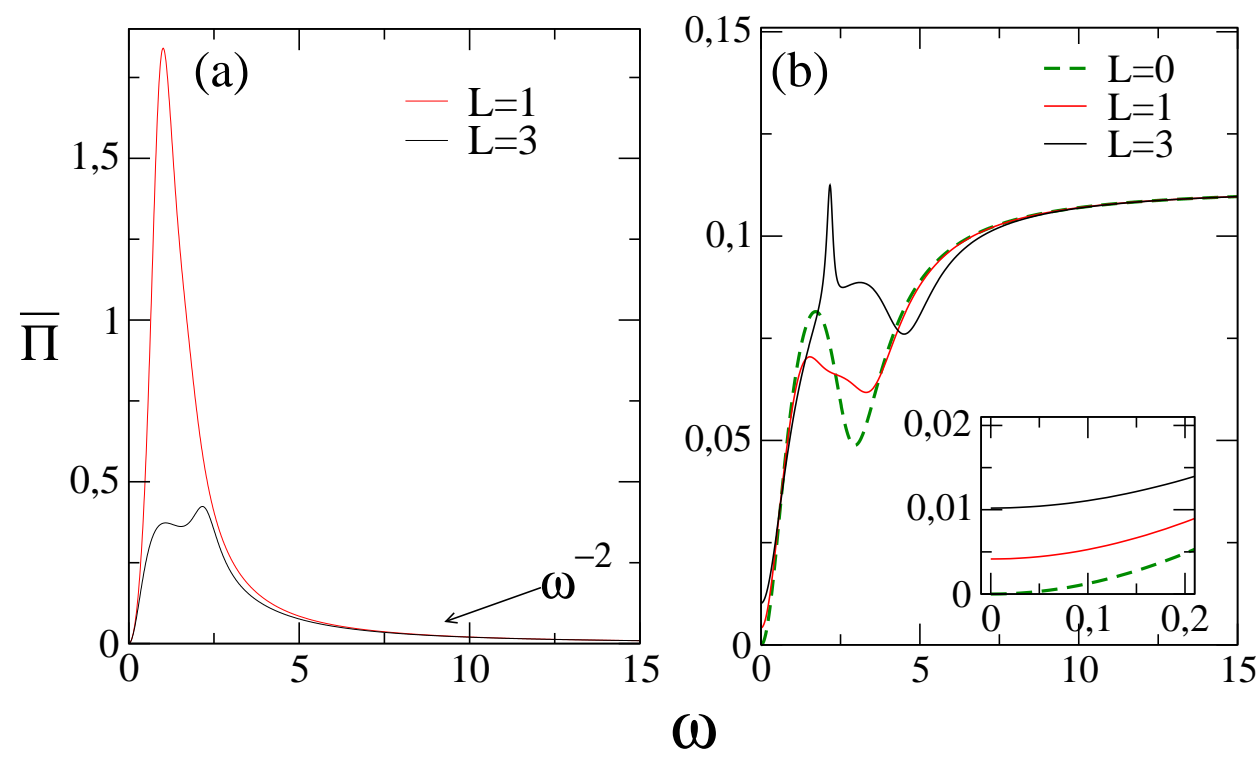

FIG. 2: Panels $(a)$ and $(b)$ depict the steady $\bar{\Pi}$ versus frequency driving $\omega$ for time dependent oscillating forces and temperatures, respectively. In all cases we take $\gamma=1, K=2, \Gamma_{1}=\Gamma_{2}=10$ and $f_{02}=2 f_{01}=4$ [panel $(a)$ ] and $F_{T_{2}}=2 F_{T_{1}}=4[$ panel (b)]. Inset: The steady $\bar{\Pi}$ for distinct $L$ 's for low $\omega$.

by

$$
\mathcal{J}_{T}=-\kappa \frac{d T}{d x}
$$

where $\kappa$ is the heat conductivity. In the case of a finite difference of temperatures $\Delta T$, it follows that $\mathcal{J}_{T} \sim 1 / N$ and thereby the heat flux is proportional to the inverse of the system size. Obtaining Fourier's Law from microscopic models have attracted great interest of in the last years [45, 46, 54, 55]. One of the simplest model consists of a chain of interacting atoms in contact with heat reservoirs at different temperatures at its ends. Among the distinct approaches aimed at obtaining a heat flux inversely proportional to the system chain, we mention the self-consistent protocol proposed by Bosterli et al. [47]. More specifically, the temperature of the $i$-th intermediate reservoir is chosen so that it does not exchange heat with the system. Here we take a somewhat different approach by Bosterli et al. in which each intermediate temperature is chosen so that the variance $\left\langle v_{i}^{2}\right\rangle_{T}$ is given by $\left\langle v_{i}^{2}\right\rangle_{T}=\Gamma_{i} /(2 \gamma)$. In contrast to Ref. [47], this selfconsistent choice leads to dissipation of heat due to the external forces and consequently all self-consistent reservoirs are expected to produce entropy.

In order to compare the distinct sources of dissipation, thermal and time oscillating forces, we will consider that particles placed at extremities are not subjected to external forces. Thereby, under the above choice the intermediate $\Gamma_{i}$ 's, the flux of entropy becomes

$$
\Phi(t)=\Phi_{T}+\Phi_{f}(t),
$$

where $\Phi_{T}$ read

$$
\Phi_{T}=\frac{2 \gamma^{2}}{\Gamma_{1}}\left\langle v_{1}^{2}\right\rangle_{T}+\frac{2 \gamma^{2}}{\Gamma_{N}}\left\langle v_{N}^{2}\right\rangle_{T}-2 \gamma
$$

and $\Phi_{f}(t)$ is a sum of individual contributions

$$
\Phi_{f}(t)=2 \gamma^{2} \sum_{i=1}^{N} \frac{\left\langle v_{i}\right\rangle^{2}}{\Gamma_{i}} .
$$

It is worth mentioning that despite the absence of external forces for extreme particles, the averages $\left\langle v_{1}\right\rangle$ and $\left\langle v_{N}\right\rangle$ present oscillating behavior due to the couplings with neighboring particles (see e.g. Eqs. (30) and (31)).

Finally, in all cases the steady entropy production rate $\Pi_{T}=\Phi_{T}$ can be written in the following form

$$
\Pi_{T}=\mathcal{J}_{T} f_{T},
$$

where the thermodynamic force $f_{T}$ and its associate flux $\mathcal{J}_{T}$ read $f_{T}=1 / \Gamma_{N}-1 / \Gamma_{1}$ and $\mathcal{J}_{T}=-\kappa\left(\Gamma_{N}-\Gamma_{1}\right) / N$, respectively. Thereby, the expression for $\Pi_{T}$ becomes

$$
\Pi_{T}=\frac{\kappa}{N} \frac{\left(\Gamma_{N}-\Gamma_{1}\right)^{2}}{\Gamma_{1} \Gamma_{N}} .
$$

Since the thermal conduction coefficient $\kappa$ is finite (it depends only on parameters $\Gamma_{1}, \Gamma_{N}, K$ and $L$ ), the entropy production $\Pi_{T}$ decays as $N^{-1}$ (see e.g. Fig. $4(b)$ ).

\section{A. Three particles}

Here we derive explicit results for a chain of $N=3$ particles. In such case, Eq. (65) becomes

$$
\Phi_{f}(t)=\frac{2 \gamma^{2}}{\Gamma_{1}}\left\langle v_{1}\right\rangle^{2}+\frac{2 \gamma^{2}}{\Gamma_{2}}\left\langle v_{2}\right\rangle^{2}+\frac{2 \gamma^{2}}{\Gamma_{3}}\left\langle v_{3}\right\rangle^{2},
$$


and the entropy production $\Pi_{T}$ due to thermal reservoirs has the shape from Eq. (66) with $\mathcal{J}_{T}$ given by

$$
\mathcal{J}_{T}=\frac{\Gamma_{1} \Gamma_{3} \gamma L^{2}\left(2 \gamma^{2} K+L^{2}\right)}{2\left[L^{2}+\gamma^{2}(4 K-2 L)\right]\left[L^{2}+\gamma^{2}(K+L)\right]} f_{T}
$$

Once again, $\Pi_{T} \geq 0$, since $4 K-2 L=2\left(k+2 k^{\prime}\right) / m$. Using the motion equations we arrive at the following expression for $\bar{\Pi}$ :

$$
\bar{\Pi}=\frac{f_{02}^{2} \gamma^{2} \omega^{2}\left[\Gamma_{1} \Gamma_{3}\left(\gamma^{2} \omega^{2}+\left(K-\omega^{2}\right)^{2}\right)+L^{2} \Gamma_{2}\left(\Gamma_{1}+\Gamma_{3}\right)\right]}{\Gamma_{1} \Gamma_{2} \Gamma_{3}\left[\gamma^{2} \omega^{2}+\left(K+2 L-\omega^{2}\right)^{2}\right]\left[\gamma^{2} \omega^{2}+\left(-K+L+\omega^{2}\right)^{2}\right]},
$$

which is strictly positive and vanishes when $f_{02}$ and/or $\omega$ are equal to zero. Also, in the regime of slow and fast oscillations, $\bar{\Pi}$ exhibit similar dependences on $\omega$ to the two particles case:

$$
\bar{\Pi} \sim\left(\frac{K^{2}}{\Gamma_{2}}+\frac{L^{2}\left(\Gamma_{1}+\Gamma_{3}\right)}{\Gamma_{1} \Gamma_{3}}\right) \frac{\gamma^{2} \omega^{2} f_{02}^{2}}{(K+2 L)^{2}(-K+L)^{2}},
$$

for $\omega<<1$ and

$$
\bar{\Pi} \sim \frac{\gamma^{2} f_{02}^{2}}{\Gamma_{2}} \frac{1}{\omega^{2}}
$$

for $\omega>>1$, respectively, implying that for such latter limit the entropy production is independent on extreme temperatures. For strong couplings between particles, $L \approx K>>\omega^{2}, \bar{\Pi}$ approaches to

$$
\bar{\Pi} \sim \frac{f_{02}^{2} \gamma^{2}}{\gamma^{2}+\omega^{2}} \frac{\left(\Gamma_{1} \Gamma_{2}+\Gamma_{1} \Gamma_{3}+\Gamma_{2} \Gamma_{3}\right)}{\Gamma_{1} \Gamma_{2} \Gamma_{3}},
$$

which, in similarity with Eq. (46) [for $N=2$ ], $\bar{\Pi}$ is independent on the interaction strengths.

\section{B. The limit of long particle chains}

All results obtained for $N=3$ particles can be straightforward extended for long chains. However, it becomes very cumbersome to obtain simplified expressions for $\bar{\Pi}$ in such cases. For this reason, we will restrict the next analysis for specific values of control parameters. Fig. 3 shows, for a chain of $N=50$ particles and three sets of temperatures $\left(\Gamma_{1}, \Gamma_{N}\right)$, the temperature profiles calculated from the self consistent protocol. In all cases, the set of intermediate temperatures changes linearly from $\Gamma_{1}$ to $\Gamma_{N}$, consistent to a flux of heat along the chain from the hot to the cold reservoirs.

Fig. 4 (a) compares the individual entropy production contributions for distinct system sizes for $f_{02}=f_{03}=$ $\ldots=f_{0 N-1}$. Since external forces are equally presented in all intermediate particles, the entropy production associated to self consistent baths increases linearly with $N$. Also, panel $(a)$ depicts the existence of two regimes. For

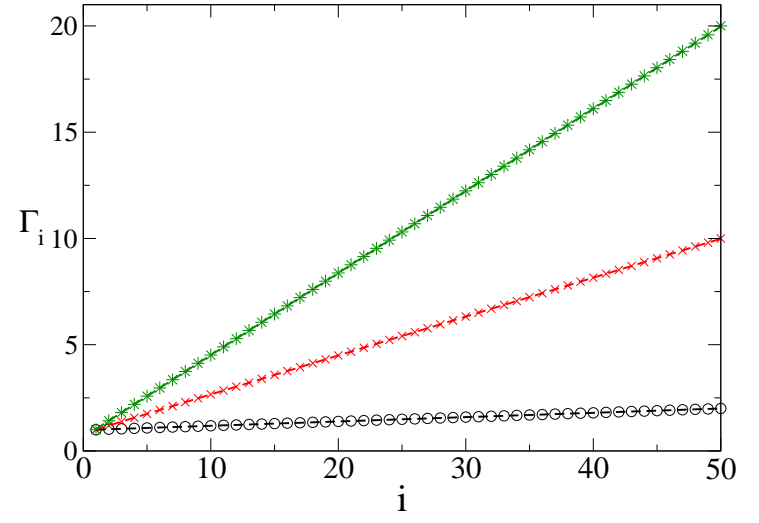

FIG. 3: For a chain of $N=50$ particles with $K=2, L=$ $1, \gamma=1$ and $\omega=1$, the rescaled temperatures $\Gamma_{i}$ 's versus the position of the $i$-th site for three set of temperatures $\left(\Gamma_{1}, \Gamma_{N}\right)$. The intermediate temperatures are calculated according to the prescription $\left\langle v_{i}^{2}\right\rangle_{T}=\Gamma_{i} /(2 \gamma)$.

small chains the thermal reservoir contribution $\Pi_{T}$ dominates over the self-consistent ones $\bar{\Pi}$, whereas $\bar{\Pi}$ wins over $\Pi_{T}$ upon $N$ is increased. In the limit $N \rightarrow \infty$ (see e.g panel $(b)$ ), only the contributions from self-consistent reservoirs prevail, in consonance with Fourier's law, Eq. (67). Finally, it is worth emphasizing two distinct linear behaviors of $\bar{\Pi}$. It arises from the particles closer to the thermal reservoirs providing more contribution for the entropy production for small chains than for large ones.

\section{CONCLUSIONS}

The nonequilibrium properties of linear chains of Brownian particles were analyzed via stochastic thermodynamics. Expressions for the heat flux, entropy production and allied quantities were exactly obtained. The regimes of short and long chains were detailed inspected. In the former case the entropy production was derived as bilinear functions of fluxes and forces, from which the associated Onsager coefficients depend on interaction couplings and frequency drivings. Reciprocal relations were also obtained. The limit of long chains was studied by 

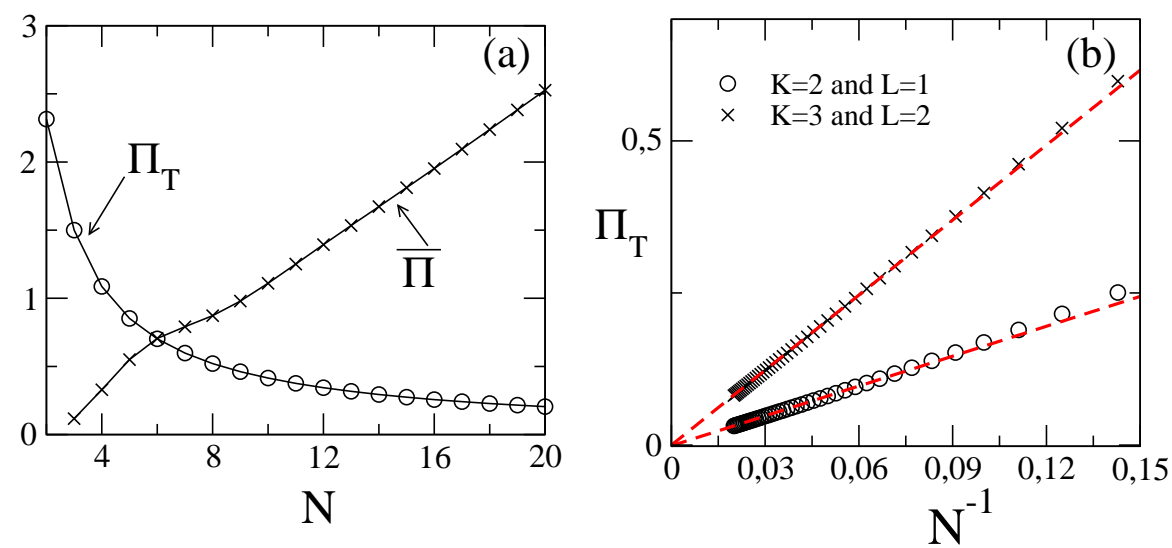

FIG. 4: For $\Gamma_{1}=1$ and $\Gamma_{N}=10$, panels $(a)$ depicts the individual entropy production contributions from the thermal and self-consistent baths versus $N$ for $K=3, L=2$ and $\omega=1.5$. In $(b)$ the behavior of entropy production from the thermal reservoirs $\Pi_{T}$ vs $N^{-1}$.

means of a self-consistent protocol for choosing intermediate temperatures. The entropy production is a sum of two terms: one coming from the real baths and the other from the self-consistent reservoirs. Whenever the former dominates for short chains, the latter contribution prevails for long ones. The contribution from the thermal reservoirs is responsible to heat flux according to Fourier's law.

As a final comment, it is worth to discuss future extensions of the present study. The inclusion of both temperature and external force drivings in harmonic chains should be interesting, in order to compare not only the structure of entropy production but also the Onsager coefficients. Also, the investigation of other kinds of drivings, such as the time discrete drivings should also be interesting in order to compare with sinusoidally time dependent ones.

\section{ACKNOWLEDGMENT}

We acknowledge Karel Proesmans for a careful reading of the manuscript. C. E. F acknowledges the financial support from FAPESP under grant 2018/02405-1.

\section{Appendix A: Obtaining the coefficients $A_{i j}$ for $N=2$ particles case with no phase difference}

Here we show explicit expressions for the coefficients $A_{i j}$ 's for the two particles case subject to oscillating forces. The index $i$ stands for the $i$-th particle $(i=1,2)$.

$$
A_{1 i}=\frac{f_{0 i}\left(K-\omega^{2}\right)\left[\gamma^{2} \omega^{2}+\left(K-\omega^{2}\right)^{2}-L^{2}\right]+f_{0 j} L\left[-\omega^{2}\left(\gamma^{2}+2 K\right)+K^{2}-L^{2}+\omega^{4}\right]}{\left[\gamma^{2} \omega^{2}+\left(K+L-\omega^{2}\right)^{2}\right]\left[\gamma^{2} \omega^{2}+\left(-K+L+\omega^{2}\right)^{2}\right]}
$$

and

$$
A_{2 i}=\frac{\gamma \omega\left[f_{0 i}\left(\gamma^{2} \omega^{2}+\left(K-\omega^{2}\right)^{2}+L^{2}\right)+2 f_{0 j} L\left(K-\omega^{2}\right)\right]}{\left(\gamma^{2} \omega^{2}+\left(K+L-\omega^{2}\right)^{2}\right)\left(\gamma^{2} \omega^{2}+\left(-K+L+\omega^{2}\right)^{2}\right)} .
$$

Having the $A_{i j}$ 's, the steady entropy production $\bar{\Pi}$ is straightforwardly evaluated.

\section{Appendix B: Obtaining the coefficients $B_{i j}$ for $N=2$ particles case and phase difference}

Here we show explicit expressions for the coefficients $B_{i j}$ 's for the two particles subject to phase difference between oscillating forces. 


$$
B_{11}=\frac{f_{02} L\left((\cos \phi-1)\left(-\omega^{2}\left(\gamma^{2}+2 K\right)+K^{2}-L^{2}+\omega^{4}\right)+2 \gamma \omega\left(K-\omega^{2}\right) \sin \phi\right)}{\left(\gamma^{2} \omega^{2}+\left(K+L-\omega^{2}\right)^{2}\right)\left(\gamma^{2} \omega^{2}+\left(-K+L+\omega^{2}\right)^{2}\right)},
$$

$$
B_{21}=\frac{f_{02} L\left(\sin \phi\left(\gamma^{2} \omega^{2}-\left(K-\omega^{2}\right)^{2}+L^{2}\right)+2 \gamma \omega\left(K-\omega^{2}\right)(\cos \phi-1)\right)}{\left(\gamma^{2} \omega^{2}+\left(K+L-\omega^{2}\right)^{2}\right)\left(\gamma^{2} \omega^{2}+\left(-K+L+\omega^{2}\right)^{2}\right)},
$$

$$
B_{12}=\frac{f_{02} \gamma \omega \sin \phi\left(\gamma^{2} \omega^{2}+\left(K-\omega^{2}\right)^{2}+L^{2}\right)+f_{02}\left(K-\omega^{2}\right)(\cos \phi-1)\left(\gamma^{2} \omega^{2}+\left(K-\omega^{2}\right)^{2}-L^{2}\right)}{\left(\gamma^{2} \omega^{2}+\left(K+L-\omega^{2}\right)^{2}\right)\left(\gamma^{2} \omega^{2}+\left(-K+L+\omega^{2}\right)^{2}\right)},
$$

and

$$
B_{22}=\frac{f_{02}\left(\omega^{2}-K\right) \sin \phi\left(\gamma^{2} \omega^{2}+\left(K-\omega^{2}\right)^{2}-L^{2}\right)+f_{02} \gamma \omega(\cos \phi-1)\left(\gamma^{2} \omega^{2}+\left(K-\omega^{2}\right)^{2}+L^{2}\right)}{\left(\gamma^{2} \omega^{2}+\left(K+L-\omega^{2}\right)^{2}\right)\left(\gamma^{2} \omega^{2}+\left(-K+L+\omega^{2}\right)^{2}\right)}
$$

respectively. Note that all of them vanishes as $\phi=0$, restoring the expressions Eqs. (A1) and (A2), respectively.

\section{Appendix C: Obtaining the coefficients $A_{i}$ 's, $B_{i}$ 's and $G_{i}$ 's for $N=2$ particles case and oscillating temperature}

Here we show explicit expressions for the coefficients $A_{i}$ 's, $B_{i}$ 's and $G_{i}$ 's for the two particles case and time

$$
\begin{aligned}
& A_{0}=2304 L^{5}\left(2 \gamma^{2}+L\right) \\
& A_{1}=-128 L^{3}\left(-7 \gamma^{4}+58 \gamma^{2} L+123 L^{2}\right), \\
& A_{2}=16 L\left(8 \gamma^{6}-50 \gamma^{4} L+34 \gamma^{2} L^{2}+931 L^{3}\right),
\end{aligned}
$$




$$
\begin{aligned}
& A_{3}=4\left(-11 \gamma^{6}+78 \gamma^{4} L+319 \gamma^{2} L^{2}-1606 L^{3}\right) \\
& A_{4}=-3\left(17 \gamma^{4}+82 \gamma^{2} L-548 L^{2}\right) \\
& A_{5}=3 \gamma^{2}-214 L \\
& A_{6}=+10 \\
& B_{0}=36864 \gamma^{4} L^{7}+18432 \gamma^{2} L^{8} \\
& B_{1}=31744 \gamma^{6} L^{5}+112640 \gamma^{4} L^{6}+27648 \gamma^{2} L^{7}+36864 L^{8} \\
& B_{2}=4608 \gamma^{8} L^{3}+18176 \gamma^{6} L^{4}-77056 \gamma^{4} L^{5}+203904 \gamma^{2} L^{6}-172032 L^{7} \\
& B_{3}=128 \gamma^{10} L+608 \gamma^{8} L^{2}-6592 \gamma^{6} L^{3}+85920 \gamma^{4} L^{4}-230720 \gamma^{2} L^{5}+269824 L^{6} \\
& B_{4}=16 \gamma^{10}-64 \gamma^{8} L+4536 \gamma^{6} L^{2}-34864 \gamma^{4} L^{3}+125488 \gamma^{2} L^{4}-170496 L^{5} \\
& B_{5}=56 \gamma^{8}-792 \gamma^{6} L+8112 \gamma^{4} L^{2}-34928 \gamma^{2} L^{3}+54288 L^{4} \\
& B_{6}=73 \gamma^{6}-928 \gamma^{4} L+5120 \gamma^{2} L^{2}-9536 L^{3} \\
& B_{7}=43 \gamma^{4}-376 \gamma^{2} L+936 L^{2} \\
& B_{8}=11 \gamma^{2}-48 L \\
& B_{9}=1 \\
& G_{0}=147456 \gamma^{6} L^{6}+147456 \gamma^{4} L^{7}+36864 \gamma^{2} L^{8} \\
& G_{1}=50176 \gamma^{8} L^{4}-94208 \gamma^{6} L^{5}+262144 \gamma^{4} L^{6}-24576 \gamma^{2} L^{7}+36864 L^{8}, \\
& G_{2}=3584 \gamma^{10} L^{2}-23552 \gamma^{8} L^{3}+166400 \gamma^{6} L^{4}-323584 \gamma^{4} L^{5}+384512 \gamma^{2} L^{6}-172032 L^{7}, \\
& G_{3}=64 \gamma^{12}-768 \gamma^{10} L+14720 \gamma^{8} L^{2}-77312 \gamma^{6} L^{3}+262528 \gamma^{4} L^{4}-399872 \gamma^{2} L^{5}+269824 L^{6} \\
& G_{4}=240 \gamma^{10}-2688 \gamma^{8} L+24672 \gamma^{6} L^{2}-96960 \gamma^{4} L^{3}+200592 \gamma^{2} L^{4}-170496 L^{5}, \\
& G_{5}=348 \gamma^{8}-3504 \gamma^{6} L+20024 \gamma^{4} L^{2}-52736 \gamma^{2} L^{3}+54288 L^{4} \\
& G_{6}=245 \gamma^{6}-2064 \gamma^{4} L+7424 \gamma^{2} L^{2}-9536 L^{3}, \\
& G_{7}=87 \gamma^{4}-528 \gamma^{2} L+936 L^{2} \\
& G_{8}=15 \gamma^{2}-48 L \\
& G_{9}=1 \text {. }
\end{aligned}
$$

[1] I. Prigogine, Introduction to Thermodynamics of Irreversible Processes, 2nd ed. (Wiley, New York, 1961).
[2] S. R. de Groot and P. Mazur, Non-Equilibrium Thermo- 
dynamics (North-Holland, Amsterdam, 1962).

[3] T. Tomé and M. J. de Oliveira, Stochastic Dynamics and Irreversibility (Springer, Cham, 2015).

[4] C. Jarzynski, Phys. Rev. Lett. 78, 2690 (1997).

[5] O.-P. Saira, Y. Yoon, T. Tanttu, M. Möttönen, D. Averin and J. P. Pekola, Phys. Rev. Lett. 109, 180601 (2012).

[6] K. Proesmans and C. Van den Broeck, Europhys. Lett. 119, 20001 (2017).

[7] Andre C. Barato and Udo Seifert Phys. Rev. Lett. 114, 158101 (2015).

[8] U. Seifert, Rep. Prog. Phys. 75, 126001 (2012).

[9] T. Tomé and M. J. de Oliveira, Phys. Rev. E 91, 042140 (2015).

[10] C. Van den Broeck and M. Esposito, Phys. A 418, 6 (2015).

[11] K Proesmans, B Cleuren and C Van den Broeck, Phys. Rev. Lett. 116, 220601 (2016).

[12] C. E. F. Noa, P. E. Harunari, M. J. de Oliveira and C. E. Fiore, Phys. Rev. E 100, 012104 (2019).

[13] T. Herpich, J. Thingna and M. Esposito, Phys. Rev. X 8, 031056 (2018).

[14] T. Herpich and M. Esposito, Phys. Rev. E 99, 022135 (2019).

[15] M. Esposito and C. Van den Broeck Physical Review E 82 (1), 011143.

[16] S. Ray and A. C. Barato, Phys. Rev. E 96, 052120 (2017).

[17] T. Tomé, Braz. J. Phys. 36, 1285 (2006).

[18] T. Tomé and M. J. de Oliveira, Phys. Rev. E 82, 021120 (2010).

[19] C. E. Fiore and M. J. de Oliveira, Phys. Rev. E 99, 052131 (2019).

[20] C. Van den Broeck and M. Esposito, Phys. Rev. E 82, 011144 (2010).

[21] A. Rosas, C. Van den Broeck and K. Lindenberg, Phys. Rev. E 94, 052129 (2016).

[22] K. Proesmans and C. Van den Broeck, Chaos 27, 104601 (2017).

[23] K. Brandner, K. Saito and Udo Seifert, Phys. Rev. X 5, 031019 (2015).

[24] K. Proesmans, B. Cleuren and C. Van de Broeck, J. Stat. Mech 2016023202 (2016).

[25] K. Proesmans and C. E. Fiore, Phys. Rev. E 100, 022141 (2019).

[26] B. Cleuren and K. Proesmans, Stochastic impedance, Physica A (2019) 122789, https://doi.org/10.1016/j.physa.2019.122789.

[27] G. W. H. Höhne, W. F. Hemminger, and H.-J. Flammersheim, Differential Scanning Calorimetry (Springer, Berlin, 2003).

[28] Y. Kraftmakher, Modulation Calorimetry, Theory and
Applications (Springer, Berlin, 2004).

[29] L. P. Filippov, Int. J. Heat Mass Transfer. 9, 681-691 (1966).

[30] P. F. Sullivan and G. Seidel, Phys. Rev. 173, 679-685 (1968).

[31] H. Gobrecht, K. Hamann and G. Willers, J. Phys. E: Sci. Instrum. 4, 21 (1971).

[32] N. O. Birge and S. R. Nagel, Phys. Rev. Lett. 54, 2674 (1985).

[33] P. S. Gill, S. R. Sauerbrunn and M. Reading, Journal of Thermal Analysis 40, 931-939 (1993).

[34] J. E. K. Schawe, Thermochimica Acta 260, 1 (1995).

[35] Y.-H. Jeong, Thermochimica Acta 304/305, 67 (1997).

[36] J. E. K. Schawe, Thermochimica Acta 304/305, 119 (1997).

[37] G. W. H. Höhne, Thermochimica Acta 304/305, 121 (1997).

[38] S. L. Simon and G. B. McKenna, J. Chem. Phys. 107 8678 (1997).

[39] K. J. Jones, I. Kinshott, M. Reading, A. A. Lacey, C. Nikolopoulos, and H. M. Pollock, Thermochimica Acta 304/305, 187 (1997).

[40] H. Baur and B. Wunderlich, Journal of Thermal Analysis 54, 437 (1998).

[41] P. Claudy and J. M. Vignon, Journal of Thermal Analysis and Calorimetry 60, 333 (2000).

[42] J.-L. Garden, Thermochimica Acta 452, 85dfl (2007).

[43] J.-L. Garden and J. Richard, Thermochimica Acta 462, 57 (2007).

[44] J.-L. Garden, J. Richard and Y. Saruyama, Journal of Thermal Analysis and Calorimetry 94, 585 (2008).

[45] G. T. Landi and M. J. de Oliveira, Phys. Rev. E 87, 052126 (2013).

[46] M. J. de Oliveira, J. Phys. A 50, 335001 (2017).

[47] M. Bolsterli, M. Rich, and W. M. Visscher, Phys. Rev. A 1, 1086 (1970).

[48] R. E. Spinney and I. J. Ford, Phys. Rev. E 85, 051113 (2012).

[49] F. Zhang, L. Xu, K. Zhang, E. Wang and J. Wang, J. Chem. Phys. 137, 065102(2012).

[50] U. Seifert, Rep. Prog. Phys. 75, 126001 (2012).

[51] M. Santillan and H. Qian, Physica A 392, 123 (2013).

[52] D. Luposchainsky and H. Hinrichsen, J. Stat. Phys. 153, 828 (2013).

[53] W. Wu and J. Wang, J. Chem. Phys. 141, 105104 (2014).

[54] S. Lepri, R. Livi and A. Politi Phys. Rep. 3771 (2003).

[55] A. Dhar, Adv. Phys. 57, 457 (2008).

[56] Z. Rieder, J. L. Lebowitz and E. Lieb, J. Math. Phys. 8, 1073 (1967). 